\title{
Subregional analysis of mnemonic functions of the prefrontal cortex in the rat
}

\author{
RAYMOND P. KESNER \\ University of Utah, Salt Lake City, Utah
}

\begin{abstract}
Evidence is reviewed to support the idea that in the rat prefrontal cortex there are differential subregional functional contributions to cognitive and memory function. It is proposed that the prefrontal cortex in the rat can be fractionated in terms of functions associated with a slightly revised rule model that incorporates a domain-specific and a level-of-processing model. It is proposed that there is a hierarchy of lower order, higher order, and highest order rules that can support different subregions of the rat prefrontal cortex. The lower order rules encompass rules associated with reversal learning. The higher order rules encompass rules associated with the different domains or attributes of working memory and cross-modal set switching. The highest order rules encompass rules associated with temporal order memory and paired associate learning and performance. It appears that there is a correspondence between rats and monkeys and between rats and humans in terms of prefrontal cortex function.
\end{abstract}

In recent years, substantial evidence has accumulated for a critical role of the prefrontal cortex (PFC) in humans in mediating working memory for specific domain or attribute information, especially that involving spatial and visual object information, as well as higher order relationships, such as learning and remembering complex tasks (e.g., paired associate learning and sequence learning), remembering the temporal order of events, selection of rules and strategies, and planning, monitoring, and modifying ongoing behavior (Fuster, 1997; Goldman-Rakic, 1996; Milner, Petrides, \& Smith, 1985; Petrides, 1996; Shimamura, Janowsky, \& Squire, 1990). In humans, it is quite difficult to assess fractionation of PFC functions on the basis of lesion data, because the lesions are highly variable and often not specific to one subregion of the PFC. However, on the basis of new imaging technology, greater possibilities for determining regional specificity have emerged (D'Esposito et al., 1998; Owen, Evans, \& Petrides, 1996; Petrides, Alivisatos, Evans, \& Meyer, 1993). Nevertheless, in order to understand more clearly the functional contribution of different anatomical regions of the PFC, attempts have been made to develop animal models of the mnemonic functions of the PFC. The major emphasis has been on developing an animal model of PFC function by using monkeys, and indeed, a substantial correspondence has been found between monkeys and humans in terms of PFC mediation of working memory and higher order processing of specific attribute information (Fuster, 1997; Goldman-Rakic, 1987). Recent research with rats has revealed that the rat can also serve as

This research was supported by NIH Grant 2R01NS20771-13. Correspondence concerning this article should be addressed to R. P. Kesner, Department of Psychology, University of Utah, 390 South 1530 East, Room 502, Salt Lake City, UT 841 12-0251 (e-mail: rpkesner@behsci. utah.edu). an excellent animal model of PFC function in monkeys and humans (Kesner, 1998; Kesner \& Jackson-Smith, 1992; Kolb, 1984). The main aim of the present paper is to review evidence supporting the view that there is a correspondence between rats, monkeys, and humans in terms of mnemonic functions of the PFC, to determine whether there are differential subregional functional contributions, and to propose a theoretical framework that potentially can integrate the empirical data on the fractionation of PFC function. There is evidence of subregional functional differentiation of other behaviors, including motor, social, affective, and inhibitory behavior. These data will not be reviewed in this paper, but for excellent reviews see Kolb (1984, 1990).

\section{SUBREGIONAL DIFFERENTIATION OF THE PREFRONTAL CORTEX IN THE RAT}

One can organize the subregions of the PFC in the rat according to the schema proposed by Uylings and van Eden (1990). These subregions include the following: the medial $P F C$, which can be subdivided into a dorsal medial region including the precentral (PC) cortex, the dorsal and ventral anterior cingulate $(\mathrm{AC})$ cortices, and a ventral medial region that includes the prelimbic and infralimbic as well as the medial orbital cortices (PL-IL/MO); the lateral $P F C$, which includes the dorsal and ventral agranular insular and the lateral orbital cortices (AI/LO); and the ventral $P F C$, which includes the ventral orbital and ventrolateral orbital cortices (VLO/VO; see Figure 1).

On the basis of anatomical observations of subregional differentiation, the rat PFC is packaged differently from those of the monkey and human cortices (Preuss, 1995), even though each prefrontal cortical area in the rat, like each prefrontal cortical area in the monkey, has reciprocal connections with the medial dorsal nucleus of the thalamus (Uylings \& van Eden, 1990). It is recognized 
A

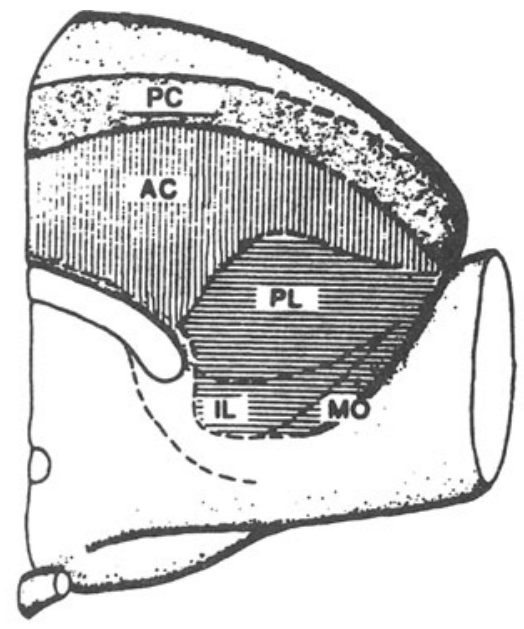

B

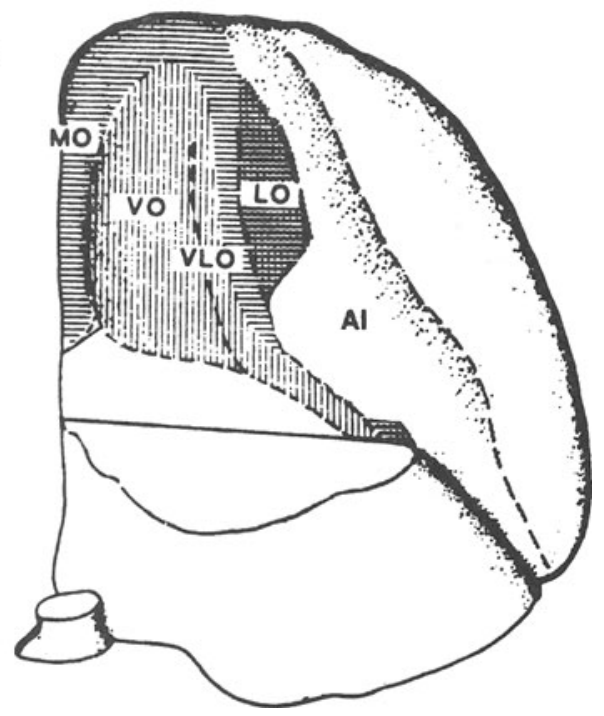

Figure 1. Schematic representation of the prefrontal cortex. (A) Medial view. (B) Ventral view. PC, precentral cortex; AC, dorsal and ventral anterior cingulate; PL-IL, prelimbic and infralimbic cortex; MO, medial orbital cortex; AI, dorsal and ventral agranular insular cortex; LO, lateral orbital cortex; VO, ventral orbital cortex; VLO, ventrolateral orbital cortex. From "The Anatomical Relationship of the Prefrontal Cortex With the Striatopallidal System, the Thalamus and the Amygdala: Evidence for Parallel Organization," by H. J. Groenewegen, H. W. Berendse, J. G. Wolters, and A. H. M. Lohman, 1990, in H. B. Uylings et al. (Eds.), The Prefrontal Cortex: Its Structure, Function, and Pathology (Progress in Brain Research, Vol. 85, p. 102). Copyright 1990 by Elsevier Science. Adapted with permission.

that a comparison of the rat PFC with the monkey and human PFCs is quite difficult. Yet, in terms of function, the same impairments that are found in monkeys with PFC lesions can also be found in rats, including learning of intramodal (reversal) and cross-modal tasks, working memory, spatial search that may involve monitoring, conditional discrimination, paired associate learning, memory for temporal order, memory for frequency tasks, and planning that uses prospective coding of information.
On the basis of Campbell and Hodos (1970) suggestion that, following lesions, one can base homologies on behavioral changes, as well as on electrophysiological recording and neural connections, I will, in addition to neural connections, draw attention to possible homologies based extensively on behavioral changes following lesions between various subregions of the rat $P F C$ and subregions of PFC in monkeys and, when possible, in humans as well.

\section{Anterior Cingulate Cortex and Precentral Cortex}

The AC cortex and the PC cortex appear to play an important role in working memory for motor responses, in memory requiring temporal processing of information, and in paired associate learning. Supporting evidence is based on the findings that lesions of the AC cortex and the PC cortex that spare the PL-IL/MO cortex produce a deficit in working memory for motor response information, such as working memory for a motor (right-left turn) response (Kesner, Hunt, Williams, \& Long, 1996), acquisition of an egocentric turn response (Kesner, Farnsworth, \& DiMattia, 1989), and acquisition of visualmotor associative conditional discriminations (Passingham, Myers, Rawlins, Lightfoot, \& Fearn, 1988; Winocur, 1991; Winocur \& Eskes, 1998). These lesions also disrupt performance associated with processing of information in complex tasks, such as memory for the temporal order of spatial information (Chiba, Kesner, \& Gibson, 1997; Chiba, Kesner, \& Reynolds, 1994; Kesner, 1998; Kesner \& Holbrook, 1987), memory for frequency information (Kesner, 1990), performance of an object-object paired associate task (Kurzina, Granholm, \& Kesner, 1995), use of a prospective code in a spatial 12 -arm working memory task (Kesner, 1989), and working memory for a list of five spatial locations (Kesner \& Holbrook, 1987).

The AC cortex and PC cortex lesions, however, do not disrupt acquisition of visual, spatial, or olfactory discrimination (Eichenbaum, Clegg, \& Feeley, 1983; Harrison \& Mair, 1996; Ragozzino, Wilcox, Raso, \& Kesner, 1999), spatial discrimination reversal, cross-modal switching from visual cue to place or from place to visual cue, switching between win-stay and win-shift rules or switching from a delayed nonmatching-to-sample to a delayed matching-to-sample rule (Harrison \& Mair, 1996; Joel, Weiner, \& Feldon, 1997; Neave, Lloyd, Sahgal, \& Aggleton, 1994; Ragozzino, Adams, \& Kesner, 1998; Wolf, Waksman, Finger, \& Almli, 1987), spatial location navigation (de Bruin, Sánchez-Santed, Heinsbroek, Donker, \& Postmes, 1994; de Bruin, Swinkels, \& de Brabander, 1997; Kesner et al., 1989; King \& Corwin, 1992), or working memory for visual object (Ennaceur, Neave, \& Aggleton, 1997; Kesner et al., 1996; Shaw \& Aggleton, 1993), duration (Jackson, Kesner, \& Amann, 1998), or affect information (DeCoteau, Kesner, \& Williams, 1997). There are also no deficits, with a few exceptions, in working memory for spatial information using delayed nonmatching to position, delayed spatial alternation, or 
nonmatching-to-sample in T-maze, 8-arm maze, and continuous spatial recognition memory procedures (Ennaceur et al., 1997; Harrison \& Mair, 1996; Joel, Tarrasch, Feldon, \& Weiner, 1997; Joel, Weiner, \& Feldon, 1997; Kesner et al., 1996; Kolb, Sutherland, \& Whishaw, 1983; Neave et al., 1994; Ragozzino et al., 1998; SánchezSanted, de Bruin, Heinsbroek, \& Verwer, 1997; Shaw \& Aggleton, 1993; Silva et al., 1986; Van Haaren, de Bruin, Heinsbroek, \& Van de Poll, 1985; Winocur, 1991; Wolf et al., 1987). Thus, the data suggest that the AC cortex and the PC process working memory for motor response information, conditioned learning with response association as an important component to be learned, and/or higher order cognitive processes, but do not process working memory for visual object, spatial, affect (taste), or time-as-duration information, as well as intramodal or cross-modal shifting of set and acquisition of spatial location navigation.

Since in the rat, $\mathrm{AC}$ cortex lesions invariably damage the $\mathrm{PC}$, it is possible that the PC cortex supports working memory for motor response information in acquiring an egocentric task, a response version in a water maze, and learning of visual response conditional tasks, whereas the AC supports visual-object-visual-object paired associate learning, temporal processing of spatial information (memory for order), memory for a list of spatial locations, and the ability to use a spatial prospective code. Supportive evidence that the PC and the AC cortex receive different pattern of inputs comes from the finding that the parietal cortex projects to the $\mathrm{PC}$, but not to the $\mathrm{AC}$ cortex, whereas the retrosplenial cortex projects to the $\mathrm{AC}$ cortex, but not to the PC cortex (Condé, Maire-Lepoivre, Audinat, \& Crépel, 1995).

On the basis of behavioral and anatomical data, the PC is assumed to be homologous to the premotor and supplementary motor areas (periarcuate or posterior dorsal lateral areas; Brodmann areas 6 and 8 ) in monkeys and humans, in that the deficits observed in the visual response conditional task and in working memory for a motor response task in rats are rather similar to what has been described for monkeys and humans. For example, in monkeys, Halsband and Passingham (1985) showed that the premotor cortex is directly involved in mediating a visual conditional motor task, but not a visual conditional nonmotor task, suggesting that the response component is critical. Similar deficits in a visual conditional response task following premotor cortex lesions have been found in monkeys and humans (Halsband \& Freund, 1990; Petrides, 1982, 1985a, 1997). Furthermore, Halsband and Freund showed that, in humans, the problem is with memory for movements and is not a problem with spatial locations. Finally, Petrides et al. (1993) have shown that the posterior dorsal lateral area is activated during the performance of a visual response conditional task. In humans with a variety of PFC lesions, Leonard and Milner (1991) showed that there is a deficit for memory of a motor movement, and Kesner, Hopkins, and Fineman
(1994) reported a memory deficit for a list of five motor movements. Further support comes from the finding that similar patterns of anatomical connections are found in rats and monkeys (Condé et al., 1995; Passingham, 1993).

On the basis of behavioral data, the $\mathrm{AC}$ cortex is assumed to be homologous to the dorsal PFC and to the dorsolateral and middorsolateral frontal cortex (Brodmann areas 9 and 9/46) in monkeys and humans, supporting visual-object-visual-object paired associate learning, temporal processing of spatial information (memory for order), memory for a list of spatial locations, self-ordering of responses, and ability to use a spatial prospective code. It should be noted that the AC cortex, which is assumed to be homologous to Brodmann areas 9 and $9 / 46$, differs from the PL-IL/MO cortex, which is assumed to be homologous with Brodmann areas 45, 46, and 47 .

In humans, in terms of memory for temporal order information, lesions of the PFC, including the dorsal PFC, result in deficits (Kesner et al., 1994; Milner, Corsi, \& Leonard, 1991; Shimamura et al., 1990). Furthermore, using fMRI, Zorrilla, Aguirre, Zarahn, Cannon, and D'Esposito (1996) showed that the dorsolateral PFC is activated in a memory for a temporal order task. In monkeys, Petrides (1991) reported that dorsolateral cortex lesions produced a deficit in memory for temporal order in formation. With respect to paired associate learning, Petrides (1985b) has shown that humans with PFC lesions have difficulty in learning a paired associate task, and Klingberg and Roland (1998) have shown, on the basis of PET scans, that the dorsolateral PFC and the ACd cortex are activated during new learning of six visual-auditory paired associates. With respect to planning, Owen (1997) has shown that lesions of the PFC in humans impair performance in a Tower of London planning task and, using PET scans during performance of the Tower of London task, that the dorsal and dorsolateral PFC is selectively activated.

\section{Prelimbic and Infralimbic Plus Medial Orbital Cortex}

The PL-IL/MO cortex appears to play an important role in working memory for visual object and spatial location information, as well as in cross-modal set switching. Supporting evidence is based on the findings that lesions of the PL-IL/MO cortex produce deficits in working memory for spatial information (Brito \& Brito, 1990; Delatour \& Gisquet-Verrier, 1996; Fritts, Ashbury, Horton, \& Isaac, 1998; Ragozinno et al., 1998; Seamans, Floresco, \& Phillips, 1995; Van Haaren et al., 1988), working memory for visual object information (Kesner et al., 1996), and cross-modal switching between place and visual cue or between visual cue and place, as well as between motor response and place and between place and motor response (Ragozzino, Detrick, \& Kesner, 1999; Ragozzino, Wilcox, et al., 1999). These lesions, however, do not affect the acquisition of spatial, motor response, or visual discriminations or of visual, motor re- 
sponse, or spatial intramodal (reversal) learning (Brito \& Brito, 1990; Bussey, Muir, Everitt, \& Robbins, 1997; Kolb et al., 1983; Ragozzino et al., 1998; Ragozzino, Detrick, \& Kesner, 1999), learning of spatial location navigation (de Bruin et al., 1994; Maaswinkel, Gispen, \& Spruijt, 1996), or working memory for affect or motor response (DeCoteau et al., 1997; Ragozzino \& Kesner, unpublished), and they produce no deficit in a visual response conditional associative task (Bussey, Muir, Everitt, \& Robbins, 1996). Thus, the data suggest that the PL-IL/MO cortex mediates working memory for spatial and visual object information and also cross-modal switching involving spatial locations or visual objects as well as spatial locations and motor responses but is not involved in motor response working memory, visual response conditional processing, or intramodal switching.

On the basis of behavioral and anatomical data, the PL-IL/MO cortex is assumed to be homologous to the dorsolateral and ventrolateral PFC area (Brodmann areas 45,46 , and 47) in monkeys and humans. Further support comes from the observation that, in rats, the posterior parietal cortex projects to the MO cortex (part of the PL-IL complex), area TE2 projects to the IL cortex, and the subiculum projects to the PL, IL, and MO cortex (Jay \& Witter, 1991; Kolb, 1990; Reep, Chandler, King, \& Corwin, 1994; Reep, Corwin, \& King, 1996). Similarly, in monkeys, the parietal cortex and subiculum project to the dorsolateral PFC and the inferotemporal cortex projects to the ventrolateral PFC (Cavada \& Goldman-Rakic, 1989; Webster, Bachevalier, \& Ungerleider, 1994). The observations with lesions of the PL-IL/MO cortex are similar to what has been reported for monkeys and humans with dorsolateral or ventrolateral PFC lesions. For example, in monkeys, lesions of the dorsolateral and ventrolateral regions disrupt performance on delayed response, delayed alternation, delayed occulomotor, spatial search, and visual object recognition tasks (Butters \& Pandya, 1969; Funahashi, Bruce, \& Goldman-Rakic, 1993; Fuster, Bauer, \& Jervey, 1982; Goldman \& Rosvold, 1970; Mishkin, 1957; Mishkin \& Manning, 1978; Passingham, 1985; Rao, Rainer, \& Miller, 1997; Wilson, Scalaidhe, \& Goldman-Rakic, 1993). Furthermore, for working memory, there are delay-specific cells in the dorsolateral and ventrolateral PFC in spatial tasks, such as delayed response, delayed alternation, and delayed occulomotor tasks, and in visual object delay tasks. In humans, D'Esposito et al. (1998) reported a meta-analysis of neuroimaging results, based on visual object and spatial location working memory tasks, that provided a strong case for processing of both visual object and spatial location information in working memory in both the dorsolateral and the ventrolateral PFC.

With respect to cross-modal set shifting, Dias, Robbins, and Roberts (1996) have shown that, in monkeys, lateral PFC lesions disrupt cross-modal, but not intramodal, switching, and Konishi et al. (1998) have shown that, with neuroimaging techniques, the critical area of activation in humans in a set-shifting paradigm is located between the dorsolateral and the ventrolateral PFC.

\section{Agranular Insular and Lateral Orbital Cortex}

On the basis of anatomical and behavioral data, the $\mathrm{AI} / \mathrm{LO}$ cortex appears to play an important role in working memory for affect information based on odor and taste. Supporting evidence is based on the findings that lesions of the $\mathrm{AI} / \mathrm{LO}$ cortex produce deficits in working memory for affect based on taste or odor information (DeCoteau et al., 1997; Otto \& Eichenbaum, 1992; Ragozzino \& Kesner, 1998). There is also some evidence that this region plays a role in the mediation of cross-modal associations in that many neurons within the $\mathrm{Al} / \mathrm{LO}$ region fire differentially for a cross-modal association between odors and locations (Lipton, Alvarez, \& Eichenbaum, 1999). There are also deficits in the acquisition and retention of a tactile-odor configuration task (Whishaw, Tomie, \& Kolb, 1992). However, there are mild or no significant deficits in odor discrimination or taste preferences (DeCoteau et al., 1997; Eichenbaum et al., 1983; Whishaw et al., 1992), in spatial working memory (Eichenbaum et al., 1983; Harrison \& Mair, 1996; Kolb et al., 1983; Ragozzino \& Kesner, 1998), and in learning a spatial location navigation task (Corwin, Fussinger, Meyer, King, \& Reep, 1994; Kolb et al., 1983; Nerad, Ramirez-Amaya, Ormsby, \& Bermudez-Rattoni, 1996). Also, there are no deficits in spatial discrimination or its reversal (Harrison \& Mair, 1996; Kolb et al., 1983). Analysis of singlecell recording from the AI, LO, and VLO cortices reveal that there are cells that respond primarily when the animal makes a reliable shift to perform in a go-no-go olfactory discrimination task. A few cells reverse their firing selectivity during reversal training, but the exact location of these cells within the AI, LO, and VLO cortex was not specified (Schoenbaum, Chiba, \& Gallagher, 1999). Thus, the data suggest that the AI/LO cortex mediates working memory for odor and taste information, as well as cross-modal associations with odor and other sensory modalities, but is not involved in spatial processing of information.

On the basis of anatomical, electrophysiological, and behavioral data, the $\mathrm{AI} / \mathrm{LO}$ cortex is also assumed to be homologous to the orbital frontal cortex (Brodmann area 13 ) in monkeys and humans, because this region supports processing of odor and taste information, as well as working memory for odor information. Cells within the orbital PFC will reverse their responsiveness to taste stimuli during reversal of a visual discrimination, suggesting the processing of taste and reward information (Rolls, 1998). Further support comes from the observation that, in rats and monkeys, there are major inputs based on taste and odor information, as well as reciprocal connections with the amygdala complex (Price et al., 1991; Rolls, 1998; Scott \& Yaxley, 1989; Shi \& Cassell, 1998).

Deficits in short-term memory for odors have been reported following damage to the orbital frontal cortex in hu- 
mans (Jones-Gotman \& Zatorre, 1993). Short-term memory for odors or taste information has not yet been reported following orbital frontal cortex lesions in monkeys.

\section{Ventrolateral and Ventral Orbital Cortex}

The role of the VLO/VO cortex in processing mnemonic information is not clear. For example, lesions of the VLO/VO cortex produce a deficit in learning a spatial location navigation task, but no deficit in learning a task requiring egocentric information and no deficit in working memory for spatial location information (Corwin et al.,1994; Janis \& Kesner, 1995). Because in the rat, there are major connections from the posterior parietal cortex and the medial and lateral extrastriate cortex to the VLO and VO cortex, providing both visual and spatial inputs into those regions (Reep et al., 1996), it is possible that both of these regions can engage learning and memory processes associated with both visual and spatial information. Clearly, more information is needed to understand the role of this region.

On the basis of behavioral data, the VLO/VO cortex is assumed to be homologous to the orbital frontal cortex (Brodmann area 12) in monkeys and humans. In monkeys, lesions of the orbital PFC produce deficits in visual object or pattern reversal learning, and cells within the orbital PFC will reverse their responsiveness to taste stimuli during reversal of a visual discrimination (Dias et al., 1996; Jones \& Mishkin, 1972; Rolls, 1998). However, visual or spatial discrimination reversal learning experiments have yet to be carried out in rats with lesions of this area.

Thus, there is a clear correspondence between rats and monkeys, and perhaps humans as well, in terms of function, in that the same impairments that are found in monkeys with PFC lesions can also be found in rats, including impairments of learning of intramodal (reversal) and cross-modal tasks, working memory, spatial search that may involve monitoring, conditional discrimination, paired associate learning, memory for temporal order, memory for frequency tasks, and planning that uses prospective coding of information. Furthermore, these behavioral data provide further support for the possibility of a distinct homology between various subregions of the rat PFC and subregions of the PFC in monkeys and, perhaps, humans as well.

\section{THEORETICAL VIEWS OF PREFRONTAL CORTEX FUNCTION}

\section{Domain Specificity Model}

A number of theoretical approaches have been taken by which functions of the PFC can be fractionated on the basis of differential subregional contributions. One approach has been taken by Goldman-Rakic $(1987,1996)$. She suggests that the main function of the PFC is to support working memory, defined as a specialized process by which a remembered stimulus is held "on line" to guide behavior in the absence of external cues. Further- more, she postulates a modular organization of working memory that is based on the use of different domains or attributes of information processing. This is called the domain specificity model. According to this model, the cortex surrounding the principal sulcus (dorsolateral PFC) is specialized for "on-line" processing of spatial information, whereas the inferior convexity (ventrolateral PFC) is specialized for "on-line" processing of visual object information. In addition, the ventrolateral PFC could also support other sensory domains. Support for this model is based on the observation of delay-specific cells in the dorsolateral PFC in only spatial tasks, such as delayed response, delayed alternation, and delayed occulomotor tasks, and the observation that lesions of the dorsolateral PFC disrupt performance on delayed response, delayed alternation, delayed occulomotor, and spatial search tasks (Butters \& Pandya, 1969; Funahashi et al., 1993; Goldman \& Rosvold, 1970; Mishkin, 1957; Passingham, 1985). In contrast, delay-specific cells in a visual object delay task are found in the ventrolateral PFC, and lesions in this area disrupt visual object recognition (Mishkin \& Manning, 1978; Wilson et al., 1993).

Research with rats is consistent with the GoldmanRakic model in that domain or attribute working memory fractionation can be seen following subregional lesions within the PFC in the rat. In this case, the PC supports working memory for response information, the $\mathrm{PL}-\mathrm{IL} / \mathrm{MO}$ cortex supports working memory for spatial location and visual object information, and the $\mathrm{AI} / \mathrm{LO}$ cortex supports working memory for odor and taste (affect) information (DeCoteau et al., 1997; Kesner et al., 1996; Otto \& Eichenbaum, 1992; Ragozzino et al., 1998; Ragozzino \& Kesner, 1998). Furthermore, these areas can operate independently of each other, in that the PC cortex does not mediate working memory for places, visual objects, or affect information, the PL-IL/MO cortex does not mediate affect or response information, and the $\mathrm{AI} / \mathrm{LO}$ cortex does not mediate spatial information (DeCoteau et al., 1997; Kesner et al., 1996; Ragozzino et al., 1998; Ragozzino \& Kesner, 1998). The other subregion-namely, the VLO/VO cortex - does not mediate working memory for spatial information (Janis \& Kesner, 1995). It is possible that, with more restricted lesions within the PL-IL/MO region, differences between spatial location and visual object working memory processing might emerge. Similarly, with more restricted lesions of the $\mathrm{Al} / \mathrm{LO}$ cortex, a difference might emerge between odor and taste working memory information.

A problem with the domain specificity model arises when one tests rats (1) in situations in which there is no delay component reducing the need for working memory, such as intramodal and cross-modal switching and paired associate learning, or (2) in situations in which there is an increased demand for working memory, when temporal processing or planning that uses prospective codes is required. For example, even though, for the $\mathrm{AC}$ and $\mathrm{PC}$ cortex, there are deficits in working memory for motor response, but not for spatial or visual object information, 
there are deficits in temporal processing of spatial information (memory for order), in memory for a list of spatial locations, and in the ability to use a spatial prospective code, as well as deficits in performance of a visual-objectvisual-object paired associate task (Kesner, 1989, 1998; Kesner \& Holbrook, 1987; Kesner et al., 1996; Kurzina et al., 1995). For the PL-IL/MO orbital cortex, even though there are deficits in working memory for spatial locations or visual objects, there are no deficits in reversal (intramodal) of visual object or spatial location discriminations and no deficits in learning a spatial navigation task (de Bruin et al., 1994; de Bruin et al., 1997; Kesner et al., 1989; Kesner et al., 1996; King \& Corwin, 1992; Ragozzino, Detrick, \& Kesner, 1999; Ragozzino, Wilcox, et al., 1999). Thus, there are data based on rat research that are not consistent with a domain or attribute fractionation of function for the PFC.

Problems with Goldman-Rakic's domain specificity model for working memory as the main organizing principle for the PFC have also emerged from research with monkeys and humans. First, Rao et al. (1997) have shown that one can record both spatial location and visual object information from the same cell within the dorsolateral PFC and that these cells can change very readily on the basis of the demands of the task. Second, Fuster et al. (1982) have shown that delay cells can be found in both the dorsolateral and ventrolateral PFC in a visual-visual stimulus or spatial-location-spatial-location matchingto-sample task. Third, lesions of the dorsolateral PFC produce problems with self-ordering or monitoring, decision making, temporal ordering, and planning of responses within a working memory system (Owen, 1997; Owen et al., 1996; Petrides, 1996).

\section{Level-of-Processing Model}

On the basis of the above-mentioned problems with the domain specificity model, Owen et al. (1996) and Petrides (1996) suggest a fractionation of the PFC on the basis of different levels of processing. They suggest that the dorsolateral PFC is involved in higher order manipulation of information and not only in the maintenance of information during delays, whereas the ventrolateral PFC is assumed to be associated with a lower order processing system involved primarily in maintaining information in working memory by selecting currently relevant information from long-term memory that is necessary for working memory. This model has been labeled the level-of-processing model.

D'Esposito et al. (1998) have summarized the results of a meta-analysis of 24 studies that have studied neuroimaging changes during use of working memory for visual object or spatial location tasks and have found that there are no significant differences in the activation of the dorsolateral or ventrolateral PFC during visual object or spatial location working memory tasks but that a difference appears in the activation of the ventrolateral region whenever information is maintained in working memory and a difference appears in the dorsolateral region when- ever one needs to monitor or manipulate information within working memory. These data provide strong support for the Petrides (1996) and Owen et al. (1996) levelof-processing model. Because this level-of-processing distinction has not been manipulated behaviorally in the rat following subregional lesions of the PFC, especially of the PL-IL/MO cortex, one cannot, at this time, evaluate the efficacy of this model in rats.

\section{Rule Model}

A completely different approach, based on data obtained in monkeys, was presented by Wise, Murray, and Gerfen (1996). Observing that deficits following PFC lesions are often assigned to the inability to efficiently use the appropriate rule or strategy to solve a problem or perform well on a task, they suggest that one can fractionate the contribution of at least five subregions of the PFC on the basis of rules or strategies, rather than on the basis of working memory. Furthermore, there is a hierarchy in terms of the complexity of the rules required, which they label lower order, higher order, and highest order. The five subregions include the orbitofrontal, the medial prefrontal, the dorsolateral prefrontal, the ventrolateral prefrontal, and the dorsal prefrontal cortical regions. This model can be labeled the rule model.

In this model, the orbitofrontal cortex supports lower order rules based on object valence and can be characterized by deficits in object reversal (intramodal set shift), object learning set, and object alternation. The medial PFC supports lower order rules based on place valence and can be characterized by deficits in place reversal (intramodal set shift).

The ventrolateral PFC supports higher order rules based on memory or attention to stimulus components and the application of abstract rules based on object information and can be characterized by deficits in matching or nonmatching rules for visual information, visual conditional associations, delayed object alternation, and object recognition with single object pairs, as well as cross-modal shifts. Data in support of a different level of analysis for the orbital frontal versus lateral PFC comes from a study by Dias et al. (1996), who showed, in marmoset monkeys, that lesions of the orbitofrontal region disrupted an intramodal shift, but not a cross-modal shift, whereas a lesion of the lateral PFC disrupted a cross-modal shift, but not an intramodal shift. The dorsolateral PFC supports higher order rules based on memory or attention to scene components and the application of abstract rules based on spatial information and can be characterized by deficits in matching or nonmatching rules for spatial information, spatial conditional associations, spatial delayed response, and spatial search, as well as cross-modal shifts.

The dorsal PFC supports the highest order rules based on event memory and the application of abstract rules based on events and can be characterized by deficits in temporal ordering of information, planning, paired associate learning, and classification of information across a particular dimension. In the rule model, working memory 
would be delegated to the higher order level, and the dorsolateral and ventrolateral distinction would be maintained; but in the rule model, the key is the utilization of rules or strategies that make working memory possible.

Even though there are some concerns about how one clearly defines the level of organization and distinguishes lower, higher, and highest order levels and about the difficulty of separating rules from tasks, it is proposed that the PFC in the rat can be fractionated in terms of functions associated with a slightly revised rule model that incorporates the domain-specific and the level-of-processing models. It is suggested that there is a hierarchy of lower order, higher order, and highest order rules that can support different subregions of the rat PFC. The lower order rules encompass rules associated with reversal learning. The higher order rules encompass rules associated with the different domains or attributes of working memory and cross-modal set switching. The highest order rules encompass rules associated with temporal order memory and paired associate learning and performance. It is possible that this hierarchy from lower to higher to highest order rules reflects increases in demands associated with relational complexity, where relational complexity is defined in terms of the number of independently varying dimensions that must be processed in parallel to activate rules and strategies that generate appropriate responses (Halford, Wilson, \& Phillips, 1998; Robin \& Holyoak, 1995). The working hypothesis is that the VLO/VO cortex supports lower order rules, the AI/LO cortex supports higher order rules for odors and tastes, the PL-IL/MO cortex support higher order rules for places and objects, the PC cortex perhaps supports higher order rules for motor responses, and finally the AI cortex supports the highest order rules for temporal ordering, paired associate learning, list learning, and planning that includes the use of temporal and prospective strategies.

Some support for the rule model comes from the following observations. (1) In rats, the VLO cortex does not mediate spatial working memory (Janis \& Kesner, 1995), and in marmosets, orbital prefrontal lesions do not impair cross-modal set shifting (Dias et al., 1996), which would reflect the engagement of higher order rules. (2) The AI/LO cortex mediates working memory for odor and taste information (DeCoteau et al., 1997; Otto \& Eichenbaum, 1992; Ragozzino \& Kesner, 1998) but is not involved in spatial processing of information (Corwin et al., 1994; Eichenbaum et al., 1983; Harrison \& Mair, 1996; Kolb et al., 1983; Ragozzino \& Kesner, 1998). Its role in the processing of cross-modal or the highest order rules has not yet been investigated. Also, there are no deficits in spatial discrimination or its reversal (Harrison \& Mair, 1996; Kolb et al., 1983), which would reflect the use of lower order rules. (3) The PL-IL/MO cortex mediates working memory for spatial and visual object information, as well as cross-modal switching involving spatial locations, visual objects, or spatial locations and motor responses (Brito \& Brito, 1990; Delatour \& Gisquet-Verrier, 1996; Kesner et al., 1996; Ragozzino et al., 1998; Ragozzino, Detrick, \& Kesner, 1999; Ragozzino, Wilcox, et al., 1999; Seamens et al., 1995; Van Haaren et al., 1988), but is not involved in motor response working memory, visual response conditional processing, or intramodal switching (Brito \& Brito, 1990; Bussey et al., 1997; Kolb et al., 1983; Ragozzino, Detrick, \& Kesner, 1999; Ragozzino et al., 1998). Its role in mediating the highest order rules has not yet been investigated. (4) The AC cortex and the PC cortex mediate rules associated with the processing of motor response information and/or higher order cognitive processes that make high demands on the relational information (Chiba et al., 1997; Chiba et al., 1994; Kesner, 1989, 1990, 1998; Kesner \& Holbrook, 1987; Kesner et al., 1996; Kurzina et al., 1995; Passingham et al., 1988; Winocur, 1991; Winocur \& Eskes, 1998) but do not process rules associated with working memory for visual object, spatial, affect (taste), or time-as-duration information, as well as with intramodal or cross-modal shifting of set and acquisition of spatial location navigation (de Bruin et al., 1994; de Bruin et al., 1997; DeCoteau et al., 1997; Ennaceur et al., 1997; Harrison \& Mair, 1996; Jackson et al., 1998; Joel, Tarrasch, et al., 1997; Joel, Weiner, \& Feldon, 1997; Kesner et al., 1989; Kesner et al., 1996; King \& Corwin, 1992; Kolb et al., 1983; Neave et al., 1994; Ragozzino et al., 1998; Sánchez-Santed et al., 1997; Shaw \& Aggleton, 1993; Silva et al., 1986; Van Haaren et al., 1985; Winocur, 1991; Wolf et al., 1987). Since in the rat, $\mathrm{AC}$ cortex lesions invariably damage the $\mathrm{PC}$, it is possible that the PC cortex supports working memory for motor response information in acquiring an egocentric task, a response version in a water maze, and learning of visual response conditional tasks and, thus, may be part of the higher order level, whereas the $\mathrm{AC}$ cortex supports visual-object-visual-object paired associate learning, temporal processing of spatial information (memory for order), memory for a list of spatial locations, and ability to use a spatial prospective code and, thus, may be a part of the highest order level.

In general, the evidence is consistent with the working hypothesis that, perhaps, the VLO/VO cortex supports lower order rules, the AI/LO cortex supports higher order rules for odors and tastes, the PL-IL/MO cortex supports higher order rules for places and objects, the PC cortex may support higher order rules for responses, and finally the AC cortex supports the highest order rules for temporal ordering, paired associate learning, list learning, and planning that includes the use of temporal and prospective strategies. Furthermore, it is encouraging that a similar level of organization applies to monkeys and, perhaps, humans as well.

\section{REFERENCES}

Brito, G. N. O., \& Brito, L. S. O. (1990). Septohippocampal system and the prelimbic sector of frontal cortex: A neuropsychological battery analysis in the rat. Behavioural Brain Research, 36, 127-146.

Bussey, T. J., Muir, J. L., EveritT, B. J., \& Robbins, T. W. (1996). Dissociable effects of anterior and posterior cingulate cortex lesions on 
the acquisition of a conditional visual discrimination: Facilitation of early learning vs. impairment of late learning. Behavioural Brain Research, 82, 45-56.

Bussey, T. J., Muir, J. L., Everitt, B. J., \& Robbins, T. W. (1997). Triple dissociation of anterior cingulate, posterior cingulate, and medial frontal cortices on visual discrimination tasks using a touchscreen testing procedure for the rat. Behavioral Neuroscience, 111, 920-936.

BUtTERS, N., \& PANDYA, D. (1969). Retention of delayed alternation: Effect of selective lesions of sulcus principalis. Science, 165, 1271 1273.

Campbell, C. B. G., \& Hodos, W. (1970). The concept of homology and the evolution of the nervous system. Brain, Behavior \& Evolution, 3, 353-367.

Cavada, C., \& Goldman-Rakic, P. S. (1989). Posterior parietal cortex in Rhesus monkey: II. Evidence for segregated corticocortical networks linking sensory and limbic areas with the frontal lobe. Journal of Comparative Neurology, 287, 422-445.

Chiba, A. A., Kesner, R. P., \& Gibson, C. J. (1997). Memory for temporal order of new and familiar spatial location sequences: Role of the medial prefrontal cortex. Learning \& Memory, 4, 311-317.

Chiba, A. A., Kesner, R. P., \& ReYNolds, A. M. (1994). Memory for spatial location as a function of temporal lag in rats: Role of hippocampus and medial prefrontal cortex. Behavioral \& Neural Biology, 61, 123-131.

Condé, F., Majre-Lepoivre, E., Audinat, E., \& Crépel, F. (1995). Afferent connections of the medial prefrontal cortex of the rat: II. Cortical and sub-cortical afferents. Journal of Comparative Neurology, 352, 567-593.

CoRwin, J. V., Fussinger, M., Meyer, R. C., King, V. R., \& ReeP, R. L. (1994). Bilateral destruction of the ventrolateral orbital cortex produces allocentric but not egocentric spatial deficits in rats. Behavioural Brain Research, 61, 79-86.

de Bruin, J. P. C., Sánchez-Santed, F., Heinsbroek, R. P. W., Donker, A., \& Postmes, P. (1994). A behavioural analysis of rats with damage to the medial prefrontal cortex using the Morris water maze: Evidence for behavioural flexibility, but not for impaired spatial navigation. Brain Research, 652, 323-333.

de Bruin, J. P. C., Swinkels, W. A. M., \& deBrabander, J. M. (1997). Response learning of rats in a Morris water maze: Involvement of the medial prefrontal cortex. Behavioural Brain Research, 85, 47-55.

DeCoteau, W. E., Kesner, R. P., \& Williams, J. M. (1997). Shortterm memory for food reward magnitude: The role of the prefrontal cortex. Behavioural Brain Research, 88, 239-249.

Delatour, B., \& Gisquet-Verrier, P. (1996). Prelimbic cortex specific lesions disrupt delayed-variable response tasks in the rat. Behavioral Neuroscience, 110, 1282-1298.

D'Esposito, M., Aguirre, G. K., Zarahn, E., Ballard, D., Shin, R. K., \& LEASE, J. (1998). Functional MRI studies of spatial and nonspatial working memory. Cognitive Brain Research, 7, 1-13.

Dias, R., Robiins, T. W., \& RoberTs, A. C. (1996). Dissociation in prefrontal cortex of affective and attentional shifts. Nature, 380, 69-72.

Eichenbaum, H., Clegg, R. A., \& Feeley, A. (1983). Reexamination of functional subdivisions of the rodent prefrontal cortex. Experimental Neurology, 79, 434-451.

EnNaCeur, A., Neave, N., \& AgGleton, J. P. (1997). Spontaneous object recognition and object location memory in rats: The effects of lesions in the cingulate cortices, the medial prefrontal cortex, the cingulum bundle and the fornix. Experimental Brain Research, 113, 509-519.

Fritts, M. E., Ashbury, E. A., Horton, J. E., \& IsaAc, W. L. (1998). Medial prefrontal lesion deficits involving or sparing the prelimbic area in the rat. Physiology \& Behavior, 64, 373-380.

Funahashi, S., Bruce, C. J., \& Goldman-Rakic, P. S. (1993). Dorsolateral prefrontal lesions and oculomotor delayed response performance: Evidence for mnemonic "scotomas." Neuroscience, 13, 14791497.

FUSTER, J. M. (1997). The prefrontal cortex: Anatomy, physiology, and neuropsychology of the frontal lobe (3rd ed.). New York: LippincottRaven.

Fuster, J. M., Bauer, R. H., \& Jervey, J. P. (1982). Cellular discharge in the dorsolateral prefrontal cortex of the monkey in cognitive tasks. Cerebral Cortex, 4, 443-450.

Goldman, P. S., \& Rosvold, H. E. (1970). Localization of function within the dorsolateral prefrontal cortex of the rhesus monkey. Experimental Neurology, 27, 291-304.

Goldman-RAKIC, P. S. (1987). Circuitry of primate prefrontal cortex and regulation of behavior by representational memory. In F. Plum \& V. Mountcastle (Eds.), Handbook of physiology: Section 1. The nervous system: Vol. 5. Higher functions of the brain (pp. 373-417). Bethesda, MD: American Physiological Society.

GoldMAN-RAKIC, P. S. (1996). The prefrontal landscape: Implications of functional architecture for understanding human mentation and the central executive. Philosophical Transactions of the Royal Society of London, 351, 1445-1453.

Groenewegen, H. J., Berendse, H. W., Wolters, J. G., \& Lohman, A. H. M. (1990). The anatomical relationship of the prefrontal cortex with the striatopallidal system, the thalamus and the amygdala: Evidence for parallel organization. In H. B. M. Uylings et al. (Eds.), The prefrontal cortex: Its structure, function, and pathology (Progress in Brain Research, Vol. 85, pp. 95-118). Amsterdam: Elsevier.

Halford, G. S., Wilson, W. H., \& Phillips, S. (1998). Processing capacity defined by relational complexity: Implications for comparative, developmental, and cognitive psychology. Behavioral \& Brain Sciences, 21, 803-864.

Halsband, U., \& Freund, H. J. (1990). Premotor cortex and conditional motor learning in man. Brain, 113, 207-222.

Halsband, U., \& Passingham, R. E. (1985). Premotor cortex and the conditions for movement in monkeys (Macaca mulatta). Behavioural Brain Research, 18, 269-276.

HaRrison, L. M., \& MaIR, R. G. (1996). A comparison of the effects of frontal cortical and thalamic lesions on measures of spatial learning and memory in the rat. Behavioural Brain Research, 75, 195-206.

JaCkson, P. A., Kesner, R. P., \& Amann, K. (1998). Memory for duration: Role of hippocampus and medial prefrontal cortex. Neurobiology of Learning \& Memory, 70, 328-348.

JANIS, T., \& KESNER, R. P. (1995). A neural system analysis of spatial recognition memory: Role of the prefrontal cortex. Society for Neuroscience Abstract, 21, 1449.

JAY, T. M., \& WITTER, M. P. (1991). Distribution of hippocampal CA1 and subicular efferents in the prefrontal cortex of the rat by means of anterograde transport of Phaseolus vulagaris-leucoagglutin. Journal of Comparative Neurology, 313, 574-586.

Joel, D., Tarrasch, R., Feldon, J., \& Weiner, I. (1997). Effects of electrolytic lesions of the medial prefrontal cortex or its subfields on 4-arm baited, 8-arm radial maze, two-way active avoidance and conditioned fear tasks in the rat. Brain Research, 765, 37-50.

Joel, D., Weiner, I., \& Feldon, J. (1997). Electrolytic lesions of the medial prefrontal cortex in rats disrupt performance on an analog of the Wisconsin Card Sorting Test, but do not disrupt latent inhibition: Implications for animal models of schizophrenia. Behavioural Brain Research, 85, 187-201.

JoNes, B., \& Mishin, M. (1972). Limbic lesions and the problem of stimulus-reinforcement associations. Experimental Neurology, 36, 362-377.

Jones-Gotman, M., \& Zatorre, R. J. (1993). Odor recognition memory in humans: Role of right temporal and orbitofrontal regions. Brain \& Cognition, 22, 182-198.

KESNER, R. P. (1989). Retrospective and prospective coding of information: Role of the medial prefrontal cortex. Journal of Experimental Brain Research, 74, 163-167.

KESNER, R. P. (1990). Memory for frequency in rats: Role of the hippocampus and medial prefrontal cortex. Behavioral \& Neural Biology, 53, 402-410.

KESNER, R. P. (1998). Neural mediation of memory for time: Role of the hippocampus and medial prefrontal cortex. Psychonomic Bulletin \& Review, 5, 585-596.

Kesner, R. P., Farnsworth, G., \& DiMattia, B. V. (1989). Doubledissociation of egocentric and allocentric space following medial prefrontal and parietal cortex lesions in the rat. Behavioral Neuroscience, 103, 956-961. 
Kesner, R. P., \& Holbrook, T. (1987). Dissociation of item and order spatial memory in rats following medial prefrontal cortex lesions. Neuropsychologia, 25, 653-664.

Kesner, R. P., Hopkins, R. O., \& Fineman, B. (1994). Item and order dissociation in humans with prefrontal cortex damage. Neuropsychologia, 32, 881-891.

Kesner, R. P., Hunt, M. E., Williams, J. M., \& Long, J. M. (1996). Prefrontal cortex and working memory for spatial response, spatial location, and visual object information in the rat. Cerebral Cortex, 6, 311 318

KeSner, R. P., \& JACKSON-Smith, P. (1992). Neurobiology of an attribute model of memory: Role of prefrontal cortex. In I. Gormezano \& E. A. Wasserman (Eds.), Learning and memory: Behavioral and biological processes (pp. 251-273). Hillsdale, NJ: Erlbaum.

KING, V. R., \& CoRwIN, J. V. (1992). Spatial deficits and hemispheric asymmetries in the rat following unilateral and bilateral lesions of posterior parietal or medial agranular cortex. Behavioural Brain Research, 50, 53-68.

KLINGBERG, T., \& Roland, P. E. (1998). Right prefrontal activation during encoding, but not during retrieval, in a non-verbal pairedassociates task. Cerebral Cortex, 8, 73-79.

KolB, B. (1984). Functions of the frontal cortex of the rat: A comparative review. Brain Research Reviews, 8, 65-98.

KoLB, B. (1990). Animal models for human PFC-related disorders. In H. B. M. Uylings et al. (Eds.), The prefrontal cortex: Its structure, function, and pathology (Progress in Brain Research, Vol. 85, pp. 501509). Amsterdam: Elsevier.

KolB, B., SUTherland, R. J., \& Whishaw, I. Q. (1983). A comparison of the contributions of the frontal and parietal association cortex to spatial localization in rats. Behavioral Neuroscience, 97, 13-27.

Konishi, S., Nakajima, K., Uchida, I., Kameyama, M., NaKahara, K., Sekihara, K., \& Miyashita, Y. (1998). Transient activation of inferior prefrontal cortex during cognitive set shifting. Nature Neuroscience, 1, 80-84.

Kurzina, N., Granholm, M., \& Kesner, R. P. (1995). Memory for object-object paired associates: Role of the prefrontal cortex. Society for Neuroscience Abstracts, 21, 1449.

Leonard, G., \& Milner, B. (1991). Contribution of the right frontal lobe to the encoding and recall of kinesthetic distance information. Neuropsychologia, 29, 47-58.

Lipton, P. A., Alvarez, P., \& Eichenbaum, H. (1999). Crossmodal associative memory representations in rodent orbitofrontal cortex. $\mathrm{Neu}$ ron, 22, 349-359.

Maaswinkel, H., Gispen, W. H., \& Spruist, B. M. (1996). Effects of an electrolytic lesion of the prelimbic area on anxiety-related and cognitive tasks in the rat. Behavioural Brain Research, 79, 51-59.

Milner, B., Corsi, P., \& Leonard, G. (1991). Frontal-lobe contribution to recency judgements. Neuropsychologia, 29, 601-618.

Milner, B., Petrides, M., \& Smith, M. L. (1985). Frontal lobes and temporal organisation of memory. Human Neurobiology, 4, 137-142.

MishKIN, M. (1957). Effects of small prefrontal lesions on delayed alternation in monkeys. Journal of Neurophysiology, 220, 615-622

Mishkin, M., \& ManNing, F. J. (1978) Non-spatial memory after selective prefrontal lesions in monkeys. Brain Research, 143, 313-323.

Neave, N., Lloyd, S., SAhgal, A., \& Aggleton, J. P. (1994). Lack of effect of lesions in the anterior cingulate cortex and retrosplenial cortex on certain tests of spatial memory in the rat. Behavioural Brain Research, 65, 89-101.

Nerad, L., Ramirez-Amaya, V., Ormsby, C. E., \& BermudezRATTONI, F. (1996). Differential effects of anterior and posterior insular cortex lesions on the acquisition of conditioned taste aversion and spatial learning. Neurobiology of Learning \& Memory, 66, 44-50.

Otto, T., \& Eichenbaum, H. (1992). Complementary roles of the orbital prefrontal cortex and the perirhinal-entorhinal cortices in an odor-guided delayed-nonmatching-to-sample task. Behavioral Neuroscience, 106, 762-775.

OWEN, A. M. (1997). Cognitive planning in humans: Neuropsychological, neuroanatomical and neuropharmacological perspectives. Progress in Neurobiology, 53, 431-450.

Owen, A. M., Evans, A. C., \& Petrides, M. (1996). Evidence for a two-stage model of spatial working memory processing within the lateral frontal cortex: A positron emission tomography study. Cerebral Cortex, 6, 31-38.

Passingham, R. E. (1985). Memory of monkeys (Macaca mulatta) with lesions in prefrontal cortex. Behavioral Neuroscience, 99, 3-21.

Passingham, R. [E.] (1993). The frontal lobes and voluntary action (Oxford Psychology Series No. 21). Oxford: Oxford University Press.

Passingham, R. E., Myers, C., Rawlins, N., Lightfoot, V., \& FEARN, S. (1988). Premotor cortex in the rat. Behavioral Neuroscience, 102, 101-109.

Petrides, M. (1982). Motor conditional associative learning after selective prefrontal lesions in the monkey. Behavioural Brain Research $5,407-413$

PeTrides, M. (1985a). Deficits in nonspatial conditional associative learning after periarcuate lesions in the monkey. Behavioural Brain Research, 16, 95-101.

Petrides, M. (1985b). Deficits on conditional associative learning tasks after frontal- and temporal-lobe lesions in man. Neuropsychologia, 23, 601-614.

PeTrides, M. (1991). Functional specialization within the dorsolateral frontal cortex for serial order memory. Proceedings of the Royal Society of London, 246, 299-306.

Petrides, M. (1996). Specialized systems for the processing of mnemonic information within the primate frontal cortex. Philosophical Transactions of the Royal Society of London: Series B, 351, 1455 1461.

Petrides, M. (1997). Visuo-motor conditional associative learning after frontal and temporal lesions in the human brain. Neuropsychologia, $35,989-997$.

Petrides, M., Alivisatos, B., Evans, A. C., \& Meyer, E. (1993). Dissociation of human mid-dorsolateral frontal cortex in memory processing. Proceedings of the National Academy of Sciences, 90, 873-877.

PrEUSS, T. M. (1995). Do rats have prefrontal cortex? The RoseWoolsey-Akert program reconsidered. Journal of Cognitive Neuroscience, 7, 1-24.

Price, J. L., Carmichael, S. T., Carnes, K. M., Clugnet, M.-C., Kuroda, M., \& RAY, J. P. (1991). Olfactory input to the prefrontal cortex. In J. L. Davis \& H. Eichenbaum (Eds.), Olfactory (pp. 101120). Cambridge, MA: MIT Press.

Ragozzino, M. E., AdaMS, S., \& KeSNER, R. P. (1998). Differential involvement of the dorsal anterior cingulate and prelimbic-infralimbic areas of the rodent prefrontal cortex in spatial working memory. $\mathrm{Be}$ havioral Neuroscience, 112, 293-303.

Ragozzino, M. E., Detrick, S., \& Kesner, R. P. (1999). Involvement of the prelimbic-infralimbic areas of the rodent prefrontal cortex in behavioral flexibility for place and response learning. Journal of Neuroscience, 19, 4585-4594.

Ragozzino, M. E., \& Kesner, R. P. (1998). The role of the agranular insular cortex in working memory for food reward value and allocentric space in rats. Behavioural Brain Research, 1, 103-112.

Ragozzino, M. E., Wilcox, C., Raso, M., \& Kesner, R. P. (1999). Involvement of rodent prefrontal cortex subregions in strategy switching. Behavioral Neuroscience, 113, 32-41.

RaO, S. R., Rainer, G., \& Miller, E. K. (1997). Integration of what and where in the primate prefrontal cortex. Science, 276, 821-823.

Reep, R. L., Chandler, H. C., King, V., \& Corwin, J. V. (1994). Rat posterior parietal cortex: Topography of corticocortical and thalamic connections. Experimental Brain Research, 100, 67-84.

ReEP, R. L., CoRwIN, J. V., \& KING, V. (1996). Neuronal connections of orbital cortex in rats: Topography of cortical and thalamic afferents. Experimental Brain Research, 111, 215-232.

RoBIN, N., \& HolyoaK, K. J. (1995). Relational complexity and the functions of prefrontal cortex. In M. S. Gazzaniga (Ed.), The cognitive neurosciences (pp. 987-997). Cambridge, MA: MIT Press.

RolLs, E. T. (1998). The orbitofrontal cortex. In A. C. Roberts, T. W. Robbins, \& L. Weiskrantz (Eds.), The prefrontal cortex: Executive and cognitive functions (pp. 67-86). Oxford: Oxford University Press.

Sánchez-Santed, F., de Bruin, J. P. C., Heinsbroek, R. P. W., \& VerWER, R. W. H. (1997). Spatial delayed alternation of rat in a T-maze: Effects of neurotoxic lesions of the medial prefrontal cortex and of T-maze rotations. Behavioural Brain Research, 84, 73-79.

Schoenbaum, G.. Chiba, A. A., \& Gallagher, M. (1999). Neural en- 
coding in orbitofrotal cortex and basolateral amygdala during olfactory discrimination learning. Journal of Neuroscience, 19, 1876-1884.

SCOTT, T. R., \& YAXLEY, S. (1989). Interaction of taste and ingestion. In R. H. Cagan (Ed.), Neural mechanisms in taste (pp. 147-177). Boca Raton, FL: CRC Press.

Seamans, J. K., Floresco, S. B., \& Phillips, A. G. (1995). Functional differences between the prelimbic and anterior cingulate regions of the rat prefrontal cortex. Behavioral Neuroscience, 109, 1063-1073.

Shaw, C., \& Aggleton, J. P. (1993). The effects of fornix and medial prefrontal lesions on delayed nonmatching-to-sample by rats. $B e$ havioural Brain Research, 54, 91-102.

SHI, C.-J., \& CASSELl, M. D. (1998). Cortical, thalamic, and amygdaloid connections of the anterior and posterior insular cortices. Journal of Comparative Neurology, 399, 440-468.

Shimamura, A. P., Janowsky, J. S., \& SQuire, L. R. (1990). Memory for the temporal order of events in patients with frontal lobe lesions and amnesic patients. Neuropsychologia, 28, 803-813.

Silva, M. G., Boyle, M. A., Finger, S., Numan, B., Bouzarra, A. A., \& AlMLI, C. R. (1986). Spatial mapping: Definitive disruption by hippocampal or medial prefrontal cortical damage in the rat. Neuroscience Letters, 65, 176-181.

UyLINGS, H. B. M., \& VAN EDEN, C. G. (1990). Qualitative and quantitative comparison of the prefrontal cortex in rat and in primates, including humans. In H. B. M. Uylings et al. (Eds.), The prefrontal cortex: Its structure, function, and pathology (Progress in Brain Research, Vol. 85, pp. 31-61). Amsterdam: Elsevier.

Van HaAren, F., de Bruin, J. P., Heinsbroek, R. P., \& Van de Poll, N. E. (1985). Delayed spatial response alternation: Effects of delayinterval duration and lesions of the medial prefrontal cortex on response accuracy of male and female Wistar rats. Behavioural Brain Research, 18, 41-49.
Van HaAren, F., van Zujderveld, G., van Hest, A., De Bruin, J. P., van Eden, C. G., \& van de Poll, N. E. (1988). Acquisition of conditional associations and operant delayed spatial response alternation: Effects of lesions in the medial prefrontal cortex. Behavioral Neuroscience, 102, 481-488.

Webster, M. J., Bachevalier, J., \& Ungerleider, L. G. (1994). Connections of inferior temporal areas TEO and TE with parietal and frontal cortex in macaque monkeys. Cerebral Cortex, 5, 470-483.

Whishaw, I. Q., Tomie, J., \& KolB, B. (1992). Ventrolateral prefrontal cortex lesions in rats impair the acquisition and retention of a tactileolfactory configural task. Behavioral Neuroscience, 106, 597-603.

Wilson, F. A. W., Scalaidhe, S. P. O., \& Goldman-Rakic, P. S. (1993). Dissociation of object and spatial processing domains in primate prefrontal cortex. Science, 260, 1955-1957.

WINOCUR, G. (1991). Functional dissociation of the hippocampus and prefrontal cortex in learning and memory. Psychobiology, 19, 11-20.

Winocur, G., \& Eskes, G. (1998). Prefrontal cortex and caudate nucleus in conditional associative learning: Dissociated effects of selective brain lesions in rats. Behavioral Neuroscience, 112, 89-101.

Wise, S. P., Murray, E. A., \& Gerfen, C. R. (1996). The frontal cortexbasal ganglia system in primates. Critical Reviews in Neurobiology, 103, 317-356.

Wolf, C., Waksman, D., Finger, S., \& AlmLI, C. R. (1987). Large and small medial frontal cortex lesions and spatial performance of the rat. Brain Research Bulletin, 18, 1-5.

Zorilla, L. T., Aguirre, G. K., Zarahn, E., Cannon, T. D., \& D'EspoSITO, M. (1996). Activation of the prefrontal cortex during judgments of recency: A functional MRI study. NeuroReport, 7, 2803-2806.

(Manuscript received October 4, 1999; revision accepted for publication February 7, 2000.) 